\title{
Subthreshold micropulse yellow laser treatment for nonresolving central serous chorioretinopathy
}

\author{
This article was published in the following Dove Press journal: \\ Clinical Ophthalmology \\ 3 December 2015 \\ Number of times this article has been viewed
}

\author{
Ahmed Hosni Abd Elhamid \\ Department of Ophthalmology, \\ Ain Shams University, Cairo, Egypt
}

Purpose: To report the efficacy and safety of micropulse $577 \mathrm{~nm}$ yellow laser in the treatment of nonresolving central serous chorioretinopathy (CSC) cases.

Patients and methods: The study included 15 eyes with nonresolving CSC lasting more than 3 months. All the patients were subjected to complete ophthalmic examination, in addition to contrast sensitivity measurement, fundus fluorescein angiography, and optical coherence tomography. All eyes were subjected to $577 \mathrm{~nm}$ subthreshold micropulse laser treatment, using the IQ 577 device, and followed up after 4 weeks, 2 months, 3 months, and 6 months. The outcome measures were change in best-corrected visual acuity, contrast sensitivity, subretinal fluid height, and change in macular thickness measured by optical coherence tomography.

Results: The average age of the patients was 36.4 years; eleven were males and four were females. Average duration of the leakage was 4.6 months. The mean best-corrected visual acuity measured 6 months after laser treatment was $0.85 \pm 0.097$, in comparison to $0.67 \pm 0.097$ before laser treatment (statistically significant $[\mathrm{SS}], P<0.05$ ). The mean central macular thickness before laser was $389.6 \pm 46.4 \mu \mathrm{m}$, in comparison to $263.6 \pm 24 \mu \mathrm{m}$ after 6 months (SS, $P<0.05$ ). The mean post-laser log contrast sensitivity measured using the Pelli-Robson contrast sensitivity chart was $1.73 \pm 0.14$, while the initial log contrast sensitivity was $1.48 \pm 0.28$ (SS, $P<0.05$ ).

Conclusion: Subthreshold micropulse laser treatment is an effective and safe treatment option for patients with nonresolving CSC.

Keywords: subthreshold micropulse laser, central muscular thickness, best corrected visual acuity, optical coherence tomography

\section{Introduction}

Central serous chorioretinopathy (CSC) is a disease that is characterized by serous macular detachment with or without retinal pigment epithelium (RPE) detachment. This detachment usually results from avascular leakage areas in the RPE. It is an idiopathic disease that is more prevalent in middle-aged men. Stress and corticosteroid intake are well-known precipitating factors. Most of the cases resolve spontaneously within 3 or 4 months with good visual prognosis. ${ }^{1}$ Considerable visual impairment occurs if the fluid persists for more than 3 months. ${ }^{2}$

Options for treatment include observation for spontaneous recovery, focal laser which carries a risk of scotoma, photodynamic therapy (PDT) which is not always effective; and intravitreal anti-vascular endothelial growth factor injection which has possible complications of retinal detachment or endophthalmitis. ${ }^{3}$

The IQ 577 laser (Iridex Corporation, Mountain View, CA, USA) is a solid-state, multimode laser. In micropulse mode, the laser energy is delivered in short pulses. The whole pulse duration is called a pulse envelope, which is divided into 100 micropulses, and each micropulse has on time and off time with a ratio depending on the duty cycle (the ratio between the on time and the whole micropulse on and off time). For example,
Correspondence: Ahmed Hosni Abd Elhamid

Department of Ophthalmology, Ain Shams University, Abbasia, Cairo, Egypt

Email hosni712000@yahoo.com 
for a $200 \mathrm{~ms}$ envelope which is divided into 100 micropulses, the micropulse duration on time will be $0.1-0.3 \mathrm{~ms}$ for a $5 \%$ and $15 \%$ duty cycle, respectively. This will produce an invisible reaction which is detectable only with microscopy and histology (subthreshold), which will be sufficient to produce an RPEconfined photothermal effect with sparing of the neurosensory retina. ${ }^{4}$ The inner retina will remain transparent and not involved as the temperature rise will be below the threshold for coagulation, in contrast to the conventional continuous-wave laser which leads to coagulation of the inner retina; thus, subthreshold micropulse laser can permit confluent therapeutic applications to the entire area of CSC. Yellow laser $(577 \mathrm{~nm})$ is poorly absorbed by xanthophyll pigment, so it could be used for treatment over the fovea. Being absorbed by both melanin and oxyhemoglobin, $577 \mathrm{~nm}$ laser causes less scatter during treatment and allows use of less power and shorter pulse durations. ${ }^{5}$

The purpose of this study was to report the efficacy and safety of micropulse $577 \mathrm{~nm}$ yellow laser in the treatment of nonresolving CSC cases.

\section{Patients and methods}

This is a prospective interventional noncomparative clinical study that was conducted in strict adherence to the tenets of the Declaration of Helsinki after approval by the local ethics committee. The study enrolled 15 eyes with CSC in the period from May 2014 to May 2015. The inclusion criteria included any age, any sex, and history of nonresolving CSC lasting for more than 3 months confirmed by fundus fluorescein angiography and optical coherence tomography (OCT). Those patients who had any causes of drop of vision other than CSC or patients that received any other form of treatment were excluded from the study.

All eyes underwent initial assessment by measuring the best-corrected visual acuity (BCVA) using Snellen chart, contrast sensitivity testing with the Pelli-Robson chart, fundus indirect ophthalmoscopy, and biomicroscopy using a 90 diopter lens, and fundus fluorescein angiography and OCT examination using 3D OCT 2000 (TOPCON Corporation, Tokyo, Japan) was done for all eyes.

After explanation of the disease with the possible benefits and side effects of laser procedure, written informed consent was signed by all patients. All the eyes were subjected to IQ 577 (577 nm) subthreshold micropulse laser treatment. The Area Centralis lens (laser spot size magnification 0.94) was used for all eyes. The parameters were individualized for every patient accordingly. The titration power, which is the power that gives a just-visible reaction on the nonedematous retina about two disc diameters from the center of the fovea, was first estimated, starting with $50 \mathrm{~mW}$ in continuous wave and then gradually increasing the power by increments of $10 \mathrm{~mW}$, moving to a new area each time, until a barely visible reaction was seen. After that, the mode was switched to micropulse and the power used in the treatment was triple the titration power. With a $200 \mu \mathrm{m}$ spot size, the pulse envelope duration was $200 \mathrm{~ms}$ and the micropulse duration was $0.2 \mathrm{~ms}(10 \%$ duty cycle). Variable numbers of confluent zero-spacing high-density shots guided by the TxCell pattern system were applied to the whole area of leakage according to each case using the $7 \times 7$ grid pattern derived from the TxCell pattern system. Retreatment was done for eyes with persistent leakage after 3 months.

The eyes were followed up after 4 weeks, 2 months, 3 months, and 6 months. OCT was done after 3 and 6 months. The outcome measures were change in BCVA, contrast sensitivity, change in subretinal fluid height, and macular thickness measured by OCT.

\section{Statistical analysis}

The raw data were entered into Excel spreadsheets and then transferred to the Statistical Package for the Social Sciences, version 21 (IBM Corporation, Armonk, NY, USA) for analysis. The characteristics of the patients are expressed as mean \pm standard deviation. Pre- and post-laser BCVA, central macular thickness (CMT), and contrast sensitivity were analyzed using paired Student's $t$-test.

\section{Results}

The study enrolled 15 eyes; the initial characteristics of the eyes are summarized in Table 1.

\section{Change in BCVA}

The mean final BCVA measured 6 months after laser treatment was $0.85 \pm 0.097$, in comparison to $0.67 \pm 0.097$ before laser treatment $(\mathrm{SS}, P<0.05)$. BCVA was improved in nine eyes $(60 \%)$, stable in six eyes $(40 \%)$, and worsened in zero eyes ( $0 \%)$ (Figure 1$)$.

Table I The initial characteristics of the patients before subthreshold micropulse yellow laser treatment

\begin{tabular}{|c|c|}
\hline \multicolumn{2}{|c|}{ Initial characteristics } \\
\hline Mean age & 36.4 years \\
\hline Sex & Eleven patients were males, four were females \\
\hline $\begin{array}{l}\text { Mean duration } \\
\text { of symptoms }\end{array}$ & 4.6 months \\
\hline $\begin{array}{l}\text { Source of } \\
\text { leakage }\end{array}$ & $\begin{array}{l}\text { Ten eyes had unifocal leakage and five eyes had } \\
\text { multifocal leakage }\end{array}$ \\
\hline & $\begin{array}{l}\text { Nine eyes had leakage inside the FAZ and six eyes had } \\
\text { leakage outside the FAZ }\end{array}$ \\
\hline Pre-laser BCVA & $0.67 \pm 0.097$ \\
\hline
\end{tabular}

Abbreviations: BCVA, best-corrected visual acuity; CSC, central serous chorioretinopathy; FAZ, foveal avascular zone. 


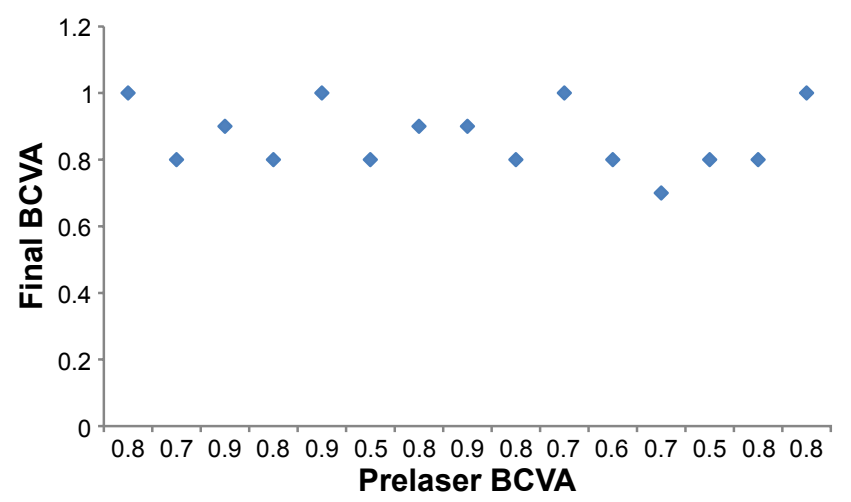

Figure I Best-corrected visual acuity (BCVA) before and 6 months after micropulse laser treatment.

\section{Change in CMT}

The mean CMT before laser was $389.6 \pm 46.4 \mu \mathrm{m}$, in comparison to $263.6 \pm 24 \mu \mathrm{m}$ after 6 months (SS, $P<0.05$ ). There was mean decrease in the CMT by $32.3 \%$ (Figure 2). Persistent submacular fluid was present in four eyes (26\%) after 3 months, for which retreatment was done. Residual minimal submacular fluid was present in two eyes after 6 months. The mean value of subretinal fluid height was $136 \mu \mathrm{m}$ before laser treatment, while it was only $10 \mu \mathrm{m} 6$ months following treatment, so it was decreased by $92.3 \%$ after treatment (Figures 3 and 4).

\section{Contrast sensitivity change}

The mean post-laser log contrast sensitivity measured by PelliRobson contrast sensitivity chart was $1.73+0.14$, while the initial $\log$ contrast sensitivity was $1.48+0.28$ (SS, $P<0.05$ ).
The average number of laser spots was $248 \pm 85$, and the average power used was $318 \pm 70.63 \mathrm{~mW}$.

\section{Discussion}

Micropulse is a laser modality that divides a continuous stream of laser into a number of short bursts separated by pauses (off time). According to the selected duty cycle, the laser stays on only $5 \%-15 \%$ of the time, thus generating less heat and preventing buildup of thermal heat with subsequent less damage to the retina than continuous-wave photocoagulation. Subthreshold micropulse laser emission without a visible burn endpoint appears to reduce the risk of structural and functional retinal laser damage, allowing treatment of foveal lesions without post-laser scotomas. It has been successfully used for diabetic macular edema and seems to be comparable to conventional argon laser. ${ }^{6}$ In 2003, Bandello et al were the first to propose subthreshold diode micropulse (SDM) photocoagulation for the treatment of CSC, showing positive results in a series of five cases. ${ }^{7}$ Ricci et al showed that SDM can provide therapeutic benefits similar to those obtainable with standard-threshold continuous-wave laser photocoagulation, but without causing discernible chorioretinal lesions, allowing almost confluent therapy and retreatment of persistent or new leaking points. ${ }^{8}$

The present study demonstrates clinical improvement in visual acuity. It also shows a decrease in macular thickness and improvement in the subretinal fluid height following subthreshold micropulse yellow laser. There was

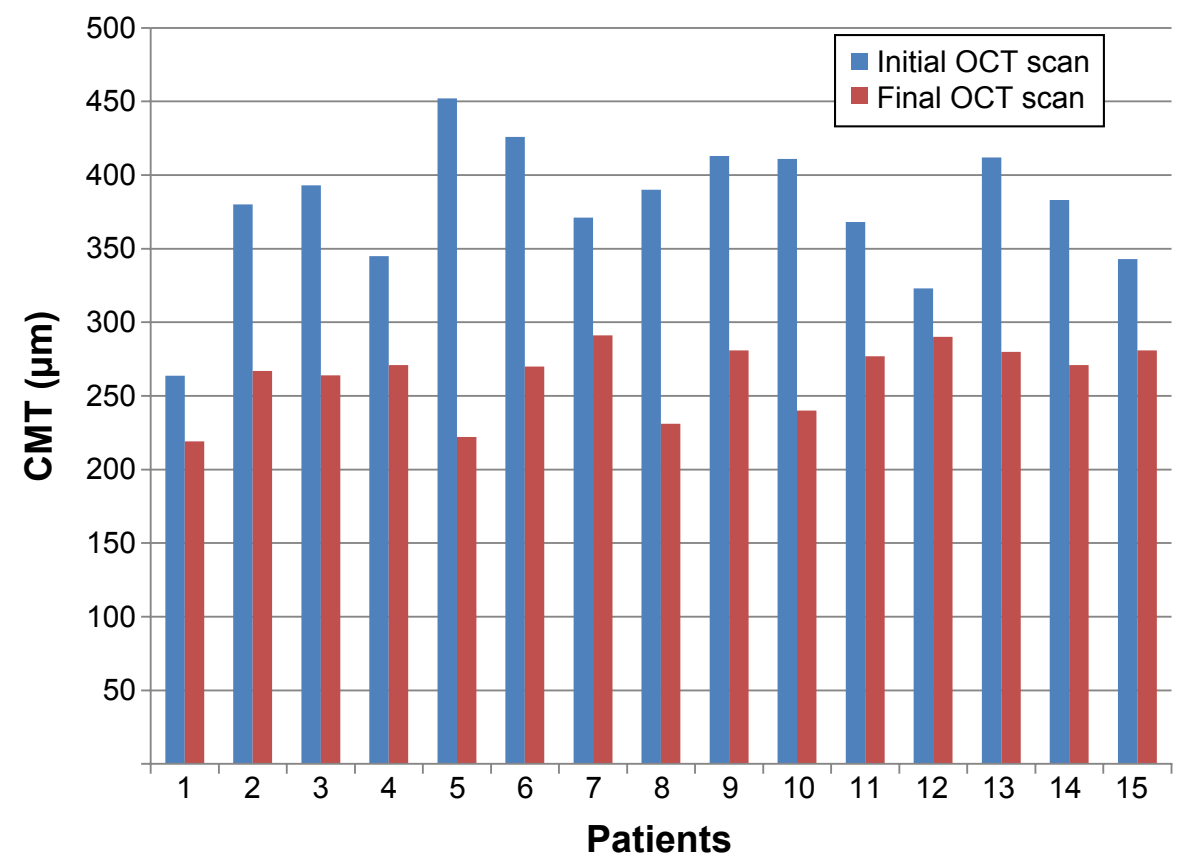

Figure 2 CMT measured before and 6 months after laser treatment for all patients.

Abbreviations: CMT, central macular thickness; OCT, optical coherence tomography. 


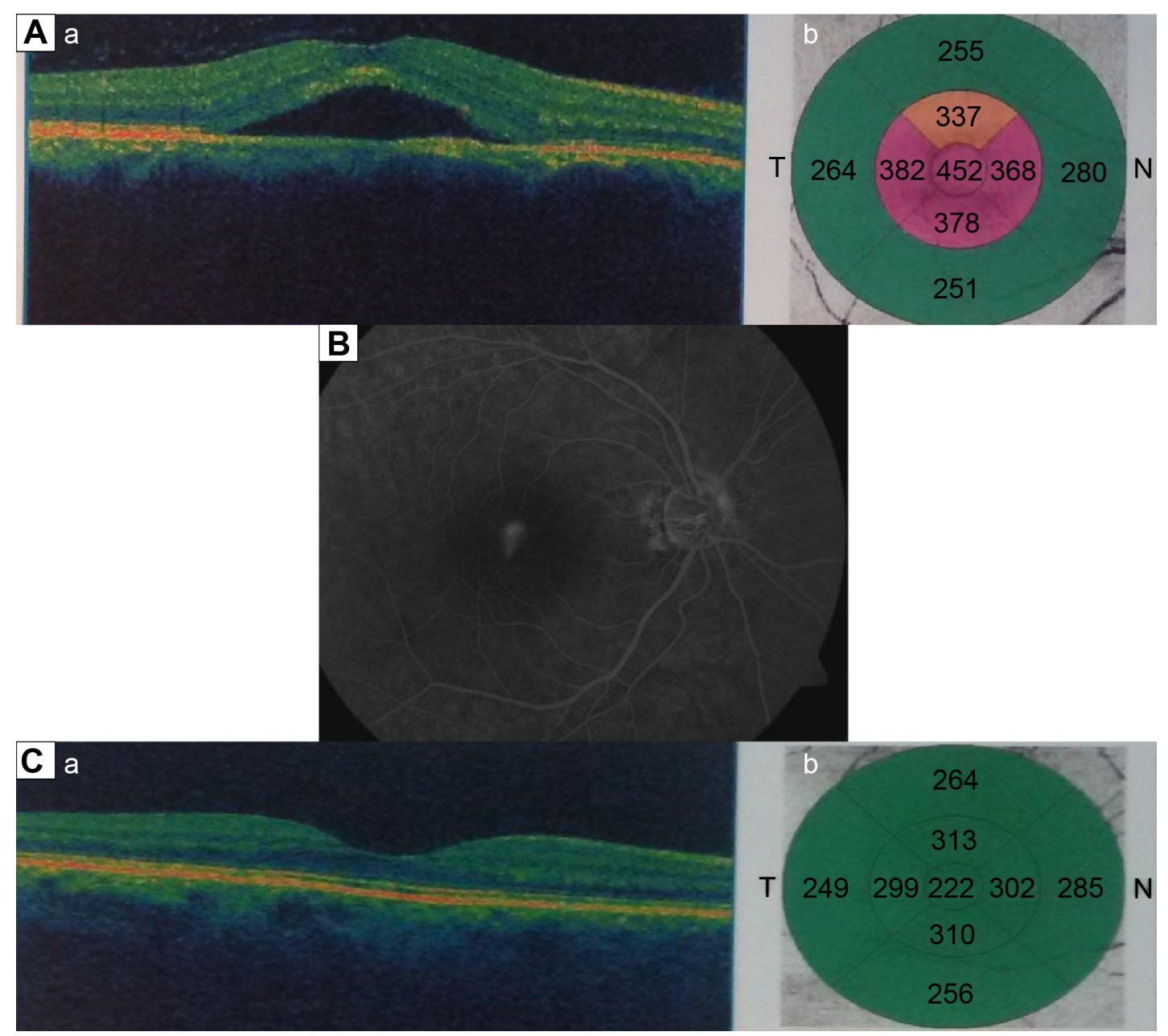

Figure $3 \mathrm{~A}$ patient with central serous chorioretinopathy of the right eye.

Notes: (A) OCT image showing neurosensory macular detachment (a) with the corresponding CMT of $452 \mu \mathrm{m}$ (b). (B) Corresponding smoke stack pattern of leakage on fundus fluorescein angiography. (C) OCT image 6 months after micropulse laser therapy with complete resolution of the fluid (a) and CMT of $222 \mu \mathrm{m}$ (b).

Abbreviations: CMT, central macular thickness; OCT, optical coherence tomography; $\mathrm{N}$, nasal; $\mathrm{T}$, temporal.

complete resolution of subretinal fluid in eleven eyes $(73 \%)$ and partial resolution in four eyes (27\%) after 3 months, and complete resolution was achieved in 13 eyes $(86.6 \%)$ after 6 months. These results are comparable to those of other studies, such as a study by Lanzetta et al, ${ }^{9}$ who reported that subretinal fluid improved in approximately $67 \%$ of cases after 1 month and in $75 \%$ at the end of follow-up. In another study by Ricci et al, ${ }^{8}$ OCT scan revealed complete resolution of subretinal fluid in five patients and a marked improvement in two patients. Chen et $\mathrm{a}^{10}$ treated 26 eyes having persistent CSC with a minimum follow-up of 6 months with complete disappearance of subretinal fluid in 55\% of eyes and partial resolution in $45 \%$ of the eyes. Yadav et a ${ }^{11}$ used subthreshold micropulse yellow laser in treating 15 eyes and reported complete resolution of subretinal fluid in $40 \%$ of the patients and partial resolution in $60 \%$ of the eyes after a follow-up period of 2 months.
BCVA was improved in eleven eyes (73.3\%), which is comparable to a study by Lanzetta et al ${ }^{9}$ that showed that BCVA was improved by one or more lines in $80.1 \%$ of eyes.

Micropulse laser causes stimulation of a biological response that restores the proper pump function of RPE cells, resulting in enhanced and rapid absorption of subretinal fluid. The $577 \mathrm{~nm}$ yellow laser is ideal for diseases in which the primary pathology is in the RPE. It is highly selective for the RPE cells and, on the other hand, it is poorly absorbed by the foveal xanthophyll pigments, hence the effects are localized to the RPE and protect the fovea.

Because yellow laser is better absorbed by the RPE and hemoglobin, it can penetrate to the RPE cells adequately to gain a similar effect with less power, which may explain the low power used in comparison to studies that used $810 \mathrm{~nm}$ diode laser (800-1,740 $\mathrm{mW}$ was used by Koss et al ${ }^{12}$ and $1,000-2,000 \mathrm{~mW}$ was used by Lanzetta et $\left.\mathrm{al}^{9}\right)$. 


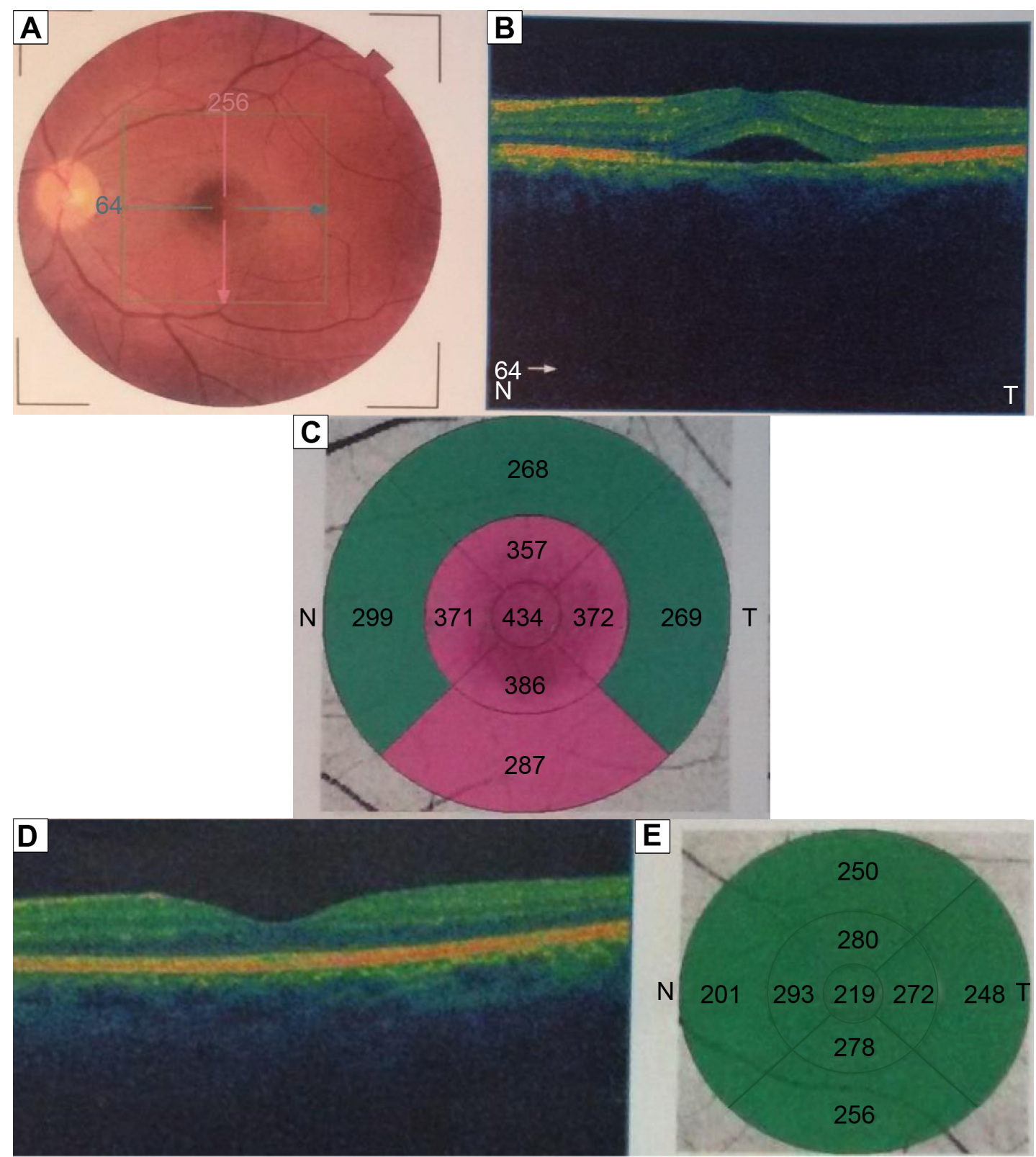

Figure $4 \mathrm{~A}$ patient with central serous chorioretinopathy of the left eye.

Notes: (A) colored image showing left CSC and (B) the corresponding OCT image, with (C) CMT of $434 \mu \mathrm{m}$. (D) OCT image after resolution of the fluid 6 months following subthreshold micropulse laser treatment, with (E) CMT $219 \mu \mathrm{m}$.

Abbreviations: CMT, central macular thickness; OCT, optical coherence tomography; $\mathrm{N}$, nasal; $\mathrm{T}$, temporal.

As noted from this study and the comparative studies discussed above, the response was relatively rapid and safe, so one may offer this modality for some groups of patients such as those with marked initial drop of vision, those with massive subretinal fluid, single-eyed patients, and those seeking rapid visual improvement due to occupational causes. Treatment at an early stage may prevent recurrences and irreversible visual loss.

To date, studies on the treatment of chronic CSC have shown that subthreshold micropulse laser can provide therapeutic benefits similar to those obtainable with standard-threshold continuous-wave laser photocoagulation, but without causing chorioretinal scars. ${ }^{11-13}$ In subthreshold laser treatment, the directly heated tissue remains viable and able to produce a stress response that induces beneficial antiangiogenic intracellular biological factors. The response of indirectly heated tissue is negligible due to minimal thermal expansion to nondirectly targeted tissue.

Thermal laser has been the historic treatment of choice for CSC and remains an option for focal leakage outside the perifoveal area. Typical parameters include a spot size of 100-200 $\mu \mathrm{m}$ for $100 \mathrm{~ms}$ at a low power of 100-200 mW. However, thermal 
laser can induce a scotoma or contrast sensitivity loss, may increase the risk of late choroidal neovascularization, and is not effective in cases with diffuse RPE decompensation and subfoveal or juxtafoveal leakage. Therefore, it is used only in selected extrafoveal cases with focal leakage. ${ }^{14}$

PDT is considered by most as one of the most important treatment lines for CSC, despite its off-label status. Yannuzzi et al first described indocyanine green-guided PDT treatment in 20 eyes, with complete resolution of fluid in $60 \%$ by 6 months. ${ }^{15}$ PDT was administered to hypercyanescent plaques (observed on mid-phase of indocyanine green angiography) at full fluence. After a 10-minute infusion of verteporfin at a dose of $6 \mathrm{mg} / \mathrm{m}^{2}$ and a wait time of $5 \mathrm{~min}$ utes, laser treatment was performed for 83 seconds at 689 $\mathrm{nm}$. However, PDT is not entirely safe and can be associated with pigmentary changes, RPE atrophy, choroidal ischemia, and secondary choroidal neovascularization. In an effort to reduce collateral damage, less intensive strategies have been employed with reduced-fluence PDT (half the exposure or half the duration of laser treatment) or reduced-dose PDT (half the dose of verteporfin). Chan et al conducted a randomized trial to compare half-dose PDT versus placebo and demonstrated that $95 \%$ of treated patients in comparison to $58 \%$ of placebo patients had complete resolution of the subretinal fluid after 1 year. Retreatment is reasonable to consider when there remains persistent fluid at least 3 months since the previous PDT, especially if there has been a positive response. ${ }^{16}$

Tseng and Chen also studied half-dose PDT in chronic CSC in 56 eyes of 56 patients. The anatomic resolution was obtained in $100 \%$ at 1 year. Four cases developed recurrence of subretinal fluid after one session of PDT. Complications included enlargement of retinal pigment epithelial atrophy in one case and choroidal neovascularization in another two cases at 12 and 14 months after PDT. ${ }^{17}$

Koss et al conducted a comparative study between SDM and intravitreal injections of bevacizumab in the treatment of chronic CSC in 52 eyes. Sixteen eyes received treatment with SDM at the active leakage site, ten eyes received an intravitreal injection of $1.25 \mathrm{mg}$ of bevacizumab, and 26 eyes underwent observation. The authors concluded that SDM photocoagulation was more effective than intravitreal injection of bevacizumab, as $12.5 \%$ of the group that received SDM therapy had persistent leakage compared with $60 \%$ of the group that received intravitreal Avastin injection after a follow-up period of 10 months. ${ }^{12}$

Behnia et al ${ }^{18}$ conducted a study on the use of subthreshold micropulse laser for cases with CSC and found significant improvement of the contrast sensitivity after laser, which was comparable also to the current study.

The greatest limitation of the SDM laser procedure is the difficulty of titrating the treatment without observing a visible reaction.

\section{Conclusion}

This trial provides evidence that subthreshold micropulse yellow laser treatment can lead to early resolution of serous retinal detachment in chronic $\mathrm{CSC}$, with significant functional improvement and without leaving any sign of laser-induced lesions. The safety of subthreshold micropulse laser enables treatment closer to the fovea over extensive areas of RPE decompensation and retreatment over the same area as needed. Further clinical studies including direct comparison with both green laser and PDT must be performed to provide more evidence-based clinical data.

\section{Disclosure}

The author reports no conflicts of interest in this work.

\section{References}

1. Spaide RF, Goldbaum M, Wong DW, Tang KC, Iida T. Serous detachment of the retina. Retina. 2003;23:820-846.

2. Guyer DR, Yannuzzi LA, Slakter JS, Sorenson JA, Ho A, Orlock D. Digital indocyanine green videoangiography of central serous chorioretinopathy. Arch Ophthalmol. 1994;112:1057-1062.

3. Ober MD, Yannuzzi LA, Do DV, et al. Photodynamic therapy for focal retinal pigment epithelial leaks secondary to central serous chorioretinopathy. Ophthalmology. 2005;112:2088-2094.

4. Luttrull JK, Spink CJ. Serial optical coherence tomography of subthreshold diode laser micropulse photocoagulation for diabetic macular edema. Ophthalmic Surg Lasers Imaging. 2006;37(5): 370-377.

5. Lanzetta P, Dorin G, Pirracchio A, Bandello F. Theoretical bases of non-ophthalmoscopically visible endpoint photocoagulation. Semin Ophthalmol. 2001;16(1):8-11.

6. Joondeph BC, Joondeph HC, Blair NP. Retinal macroaneurysms treated with the yellow dye laser. Retina. 1989;9:187-192.

7. Bandello F, Lanzetta P, Furlan F, Polito A. Non visible subthreshold micropulse diode laser treatment of idiopathic central serous chorioretinopathy. A pilot study. Invest Ophthalmol Vis Sci. 2003; 44:4858.

8. Ricci F, Missiroli F, Regine F, Grossi M, Dorin G. Indocyanine green enhanced subthreshold diode-laser micropulse photocoagulation treatment of chronic central serous chorioretinopathy. Graefes Arch Clin Exp Ophthalmol. 2009;247(5):597-607.

9. Lanzetta P, Furlan F, Morgante L, Veritti D, Bandello F. Nonvisible subthreshold micropulse diode laser $(810 \mathrm{~nm})$ treatment of central serous chorioretinopathy. A pilot study. Eur J Ophthalmol. 2008;18(6): 934-940.

10. Chen SN, Hwang JF, Tseng LF, Lin CJ. Subthreshold diode micropulse photocoagulation for the treatment of chronic central serous chorioretinopathy with juxtafoveal leakage. Ophthalmology. 2008;115(12): 2229-2234.

11. Yadav NK, Jayadev C, Mohan A, et al. Subthreshold micropulse yellow laser $(577 \mathrm{~nm})$ in chronic central serous chorioretinopathy: safety profile and treatment outcome. Eye (Lond). 2015;29(2):258-264. 
12. Koss MJ, Beger I, Koch FH. Subthreshold diode laser micropulse photocoagulation versus intravitreal injections of bevacizumab in the treatment of central serous chorioretinopathy. Eye (Lond). 2012;26(2): 307-314.

13. Cardillo JA, Daré A, Lavinsky D, Farah ME. 577 selective subthreshold laser photocoagulation for the treatment of central serous retinopathy with foveal leakage. Paper presented at: Retina Society Annual Meeting; September 21-25 2012; Rome.

14. Burumcek E, Mudun A, Karacorlu S, Arslan MO. Laser photocoagulation for persistent central serous retinopathy: results of long-term follow-up. Ophthalmology. 1997;104(4):616-622.

15. Yannuzzi LA, Slakter JS, Gross NE, et al. Indocyanine green angiographyguided photodynamic therapy for treatment of chronic central serous chorioretinopathy: a pilot study. Retina. 2003;23(3):288-298.
16. Chan WM, Lai TY, Lai RY, Liu DT, Lam DS. Half-dose verteporfin photodynamic therapy for acute central serous chorioretinopathy: oneyear results of a randomized controlled trial. Ophthalmology. 2008; 115(10):1756-1765.

17. Tseng CC, Chen SN. Long-term efficacy of half-dose photodynamic therapy on chronic central serous chorioretinopathy. $\mathrm{Br} J$ Ophthalmol. 2015;99(8):1070-1077.

18. Behnia M, Khabazkhoob M, Aliakbari S, Abadi AE, Hashemi H, Pourvahidi P. Improvement in visual acuity and contrast sensitivity in patients with central serous chorioretinopathy after macular subthreshold laser therapy. Retina. 2013;33(2):324-328.
Clinical Ophthalmology

\section{Publish your work in this journal}

Clinical Ophthalmology is an international, peer-reviewed journal covering all subspecialties within ophthalmology. Key topics include: Optometry; Visual science; Pharmacology and drug therapy in eye diseases; Basic Sciences; Primary and Secondary eye care; Patien Safety and Quality of Care Improvements. This journal is indexed on

Submit your manuscript here: http://www.dovepress.com/clinical-ophthalmology-journal

\section{Dovepress}

PubMed Central and CAS, and is the official journal of The Society of Clinical Ophthalmology (SCO). The manuscript management system is completely online and includes a very quick and fair peer-review system, which is all easy to use. Visit http://www.dovepress.com/ testimonials.php to read real quotes from published authors. 